(2) Open Access Full Text Article

\title{
Acquired latent tuberculosis infection in psoriasis patients treated with etanercept in the People's Republic of China
}

This article was published in the following Dove Press journal:

Drug Design, Development and Therapy

12 October 2015

Number of times this article has been viewed

\section{Cheng-Rang Li \\ Qiu-Xia Mao \\ Min Chen \\ Wei-Xue Jia \\ Xu Yao \\ Su-Ying Feng \\ Hong Jia \\ Juan-Qin Gong \\ Xue-Yuan Yang}

Institute of Dermatology, Chinese Academy of Medical Sciences \& Peking Union Medical College, Nanjing, Jiangsu, People's Republic of China
Correspondence: Xue-Yuan Yang Institute of Dermatology, Chinese Academy of Medical Sciences \& Peking Union Medical College, 12 jiang-Wang-Miao Street, Nanjing 210042, Jiangsu, People's Republic of China

Tel +86258547809l

Email yangxueyuan@medmail.com.cn
Background: TNF- $\alpha$ plays a key role in host defense against mycobacterial infection, and patients receiving TNF- $\alpha$ blocker treatment have increased susceptibility to tuberculosis disease. In the People's Republic of China, an intermediate tuberculosis-burden country, the latent tuberculosis infection (LTBI) risk in patients with psoriasis who are treated with etanercept, the safest kind of TNF- $\alpha$ blocker, is unknown.

Objectives: This study reports the LTBI risk in patients with psoriasis after etanercept treatment and aims to answer the question of how often rescreening for LTBI should be done in order to reduce active tuberculosis infection of patients and further reduce the incidence of active tuberculosis disease.

Patients and methods: This retrospective review evaluated patients with moderate-to-severe chronic plaque psoriasis between 2009 and 2013. All patients were excluded tuberculosis infection and received etanercept $25 \mathrm{mg}$ twice weekly, then the patients were checked for LTBI 3 months after etanercept treatment to observe the incidence of LTBI and assess the need for rescreening for LTBI every 3 months.

Results: We retrospectively analyzed 192 patients with psoriasis with moderate-to-severe chronic plaque whose tuberculin skin test and chest X-rays were negative and who received etanercept $25 \mathrm{mg}$ twice weekly. Eighteen of them were excluded because they received less than 3 months of etanercept therapy. After treatment with etanercept, four patients were found to have LTBI.

Conclusion: In this study, the incidence of LTBI after 3 months was four in 192 (2.1\%), which is higher than the annual incidence of LTBI in the People's Republic of China (0.72\%), so LTBI could be expected to occur within 3 months in psoriasis patients on etanercept. Periodic screening for LTBI in the therapy course, as well as before initiating treatment, is necessary in those patients who use a TNF- $\alpha$ blocker. We recommend rescreening for LTBI every 3 months.

Keywords: TNF receptor, TNFR, fusion protein, treatment, LTBI, TB screening

\section{Introduction}

Psoriasis is a chronic, inflammatory, systemic disease that affects approximately $2 \%$ of the world's population. ${ }^{1}$ Patients with psoriasis have more physical and mental disabilities than those with many major diseases. ${ }^{2}$ Patients with severe chronic plaque psoriasis are not satisfied with the treatment effect, because cumulative toxicity of the therapies limits their usefulness in this chronic disease. Early reports by different investigators demonstrated that tumor necrosis factor (TNF)- $\alpha$ was elevated in sera and overexpressed in skin tissue of psoriatic lesions in patients with psoriasis..$^{3,4}$ Etanercept is a bioengineered human TNF receptor (TNFR) fusion protein incorporating soluble TNFR p75 and the Fc component of IgG 1. This recombinant product 
binds specifically to TNF- $\alpha$ and lymphotoxin, inhibiting their interaction with cell-surface receptors. Etanercept has shown efficacy in the treatment of adults with psoriasis. ${ }^{5,6}$ However, as TNF- $\alpha$ plays a key role in host defense against mycobacterial infection, patients receiving such treatment have increased susceptibility to serious infections, including tuberculosis (TB). ${ }^{7,8}$ However, to date, studies of acquired latent TB infection (LTBI) of psoriasis patients treated with etanercept in the People's Republic of China has not been published. Such a study can help us to understand the LTBI risk of etanercept in Chinese patients.

LTBI is defined as a state of persistent immune response to stimulation by Mycobacterium tuberculosis antigens without evidence of clinically manifested active TB. ${ }^{9}$ However, the risk of developing TB disease following infection depends on several factors, the most important one being the immunological status of the host. A direct measurement tool for M. tuberculosis infection in humans is currently unavailable. Systematic testing and treatment of LTBI should be performed in patients initiating anti-TNF treatment. Either interferon-gamma release assays or the Mantoux tuberculin skin test (TST) should be used to test for LTBI. ${ }^{10}$ According to a national epidemiological survey of TB in 2000, the annual incidence of LTBI is $0.72 \%$ in the People's Republic of China. ${ }^{11}$

\section{Patients and methods}

\section{Patients}

We retrospectively reviewed psoriasis patients treated with etanercept between 2009 and 2013. They were outpatients and inpatients. Before and after treatment with etanercept, all patients needed to be examined to rule out TB. Patients who had clinically active TB or a positive TST or showed radiographic evidence of fibrocalcified lesions in the upper lung fields were excluded from using etanercept. As TB usually appears several months after treatment with TNF blockers, patients who used etanercept for less than 3 months were excluded from the analysis.

All patients signed written informed consents. The study was conducted in accordance with the principles of the Declaration of Helsinki and was approved by our local ethics committee, the Institutional Ethical Review Board of Peking Union Medical College.

\section{TST and T-SPOT ${ }^{\circledR}$.TB test for LTBI}

The TST was performed with an intradermal injection of two tuberculin units of purified protein derivative RT-23 (Statens Serum Institut, Copenhagen, Denmark) into the ventral surface of the forearm, according to the Mantoux method. In the People's Republic of China, a TST induration cutoff $\geq 5 \mathrm{~mm}$ is considered positive. The T-SPOT ${ }^{\circledR}$.TB test (Oxford Immunotec, Abingdon, UK), an interferongamma release assay for TB infection, does not cross-react with bacille Calmette-Guérin or most non-tuberculosis Mycobacterium spp. and is based on interferon-gamma responses to $M$. tuberculosis-specific antigens. The $\mathrm{T}_{-S P O T}{ }^{\circledR}$.TB test was conducted in patients whose TST changed to a positive result after etanercept treatment. For the $\mathrm{T}_{-S P O T}{ }^{\circledR}$.TB, a $10 \mathrm{~mL}$ blood sample was taken and analyzed according to the manufacturer's instructions. ${ }^{12,13}$

\section{Diagnosis and prophylactic treatment of LTBI}

After etanercept treatment, LTBI was confirmed when a patient's TST test changed to a positive result but they did not have any clinical, radiological, or microbiological evidence of active TB. There is a lack of consensus regarding LTBI treatment. A 3-month regimen of isoniazid $300 \mathrm{mg}$ daily plus rifampicin $450 \mathrm{mg}$ daily was recommended but not mandatory for the LTBI patients. After anti-TB treatment for 3 months, the TST and T-SPOT ${ }^{\circledR}$.TB test were conducted again.

\section{Results \\ Patients treated with etanercept}

Demographic and clinical characteristics of the study population are reported in Table 1 . We retrospectively analyzed 192 patients with psoriasis with moderate-to-severe chronic plaque psoriasis whose TST and chest X-rays were negative and who received etanercept $25 \mathrm{mg}$ twice weekly. Eighteen of them were excluded because they received less than 3 months of etanercept therapy. The patients, including a total of 141 male patients and 51 female patients, had the following characteristics: mean age 40.8 \pm 12.4 (range 16-67) years; mean height $167.5 \pm 7.2$ (range 150-185) cm; mean weight 67.1 \pm 10.4 (range 45-87) kg; mean Psoriasis Area and Severity Index (PASI) score 21.4 \pm 10.3 (range 10-53); and mean course of disease $12.2 \pm 9.8$ (range $0.5-40$ ) years. None of the

Table I Demographic and clinical characteristics of the study population

\begin{tabular}{ll}
\hline Number of patients with psoriasis & 192 \\
\hline Male (\%)/female (\%) & $141(73.4) / 51$ (26.6) \\
Mean age (years) & $40.8 \pm 12.4$ (range I6-67) \\
Mean height $(\mathrm{cm})$ & $167.5 \pm 7.2$ (range I50-185) \\
Mean weight $(\mathrm{kg})$ & $67.1 \pm 10.4$ (range 45-87) \\
Mean PASI score & $21.4 \pm 10.3$ (range I0-53) \\
Mean course of disease (years) & $12.2 \pm 9.8$ (range 0.5-40) \\
\hline
\end{tabular}

Abbreviation: PASI, Psoriasis Area and Severity Index. 
psoriasis patients systematically used immunosuppressive agents such as methotrexate, cyclosporin, and glucocorticoids, and none of them had malnutrition, immunosuppresion, HIV, or a definite history of contact with patients with TB, etc.

\section{TST and T-SPOT ${ }^{\circledR}$.TB test results after more than 3 months of continuous etanercept therapy}

After treatment with etanercept for 3 months, TSTs were performed again, and we found four patients' TSTs had become positive. TSTs were done $0-3$ days after the last dose of etanercept. Three of the four patients also had a positive $\mathrm{T}^{-S P O T}{ }^{\circledR}$.TB test. No patients with a positive TST had any clinical, radiological, or microbiological evidence of active TB, and LTBI was confirmed.

\section{Prophylactic treatment of LTBI and patient outcomes}

Three of the LTBI patients received therapy with isoniazid $300 \mathrm{mg}$ daily and rifampicin $450 \mathrm{mg}$ daily. After 3 months of anti-TB treatment, they were given the TST and T-SPOT ${ }^{\circledR}$.TB test again. One patient's screening test converted to negative and those of another two patients were improved. We suggested the two patients whose tests did not convert to negative should retest for LTBI after 6 months, but they failed to retest for LTBI for some reason. One of the LTBI patients did not receive any anti-TB treatment but stopped etanercept. This patient repeated the T-SPOT ${ }^{\circledR}$.TB test 8 months later and had a negative result.

\section{Discussion}

In 2013, the largest number of new TB cases occurred in the South East of Asia and Western Pacific regions, accounting for $56 \%$ of new cases globally. ${ }^{14,15}$ The prevalence of LTBI in the People's Republic of China is still more than $44.5 \%,{ }^{11}$ although it has shown a sustained decline over the past decade.

It is a well-established finding that use of a TNF blocker increases the risk for TB disease. Published data from registries suggest that etanercept was associated with $\mathrm{TB}$, but the incidence was lower and associated with a longer time to TB onset when compared to infliximab and adalimumab. ${ }^{16,17}$ The British Society for Rheumatology Biologics Register (BSRBR) confirms that the relative risk of TB is three to four times higher with the monoclonal antibodies than with etanercept. ${ }^{17}$

In the present study, we found that four patients acquired LTBI after 3 months of treatment with etanercept, which implies that one should recheck for TB every 3 months. LTBI is the presence of M. tuberculosis in the body with neither signs and symptoms nor radiographic or bacteriologic evidence of TB disease. It is estimated that around $10 \%$ of LTBI carriers are potentially at risk of developing an active infection, which is both symptomatic and contagious. Early detection and treatment of LTBI while on TNF-inhibitor therapy may result in better outcomes for the patient. ${ }^{18}$ The probability of developing active TB is reportedly up to seven times higher when early detection and treatment of LTBI are not followed. ${ }^{19}$ There may be several regimens of prophylactic therapy available within a single country. ${ }^{20,21}$ Nine months of isoniazid treatment is recommended by the US Centers for Disease Control and Prevention (CDC) and American Thoracic Society (ATS). ${ }^{22}$ Given the high incidence and the high multidrug resistance of TB in the People's Republic of China, LTBI patients are typically given therapy with isoniazid $300 \mathrm{mg}$ daily and rifampicin $450 \mathrm{mg}$ daily. ${ }^{23,24}$

A limitation of the present study was lack of a control group, because it was a retrospective study. In the absence of a placebo arm, conclusions about acquired LTBI are less reliable; however, the TST results of four patients in this article became positive after 3 months of treatment with etanercept. In our study, the incidence of LTBI in 3 months was four in $192(2.1 \%)$, which is higher than the annual incidence of LTBI in the People's Republic of China $(0.72 \%),{ }^{11}$ so acquired LTBI is a plausible result of using etanercept.

\section{Conclusion}

We have reported LTBI induced by mid-dose and shortcourse etanercept treatment in the People's Republic of China. Although etanercept is the safest TNF blocker, periodic screening for LTBI in the therapy course, as well as before initiating treatment, is necessary. We recommend rescreening for LTBI every 3 months. However, in order to better answer how often rescreening for LTBI should be done, we expect other authors will design further scientific prospective studies that will involve expanding the sample size; rescreening for LTBI every 1, 2, 3, 4, or 5 months; rescreening for LTBI 1-3 weeks after the last dose of etanercept; and so on.

\section{Acknowledgments}

We thank the patients for their participation in this study. This work was supported by the Foundation of Six Talent Peaks Project in Jiangsu Province (2013-WSW-060) and 
the National Natural Science Foundation of China (grant number 81472872).

\section{Disclosure}

The authors report no conflicts of interest in this work.

\section{References}

1. Koo J. Population-based epidemiologic study of psoriasis with emphasis on quality of life assessment. Dermatol Clin. 1996;14:485-496.

2. Rapp SR, Feldman SR, Exum ML, Fleischer AB Jr, Reboussin DM. Psoriasis causes as much disability as other major medical diseases. J Am Acad Dermatol. 1999;41:401-407.

3. Bonifati C, Ameglio F. Cytokines in psoriasis. Int J Dermatol. 1999; 38:241-251

4. Ettehadi P, Greaves MW, Wallach D, Aderka D, Camp RD. Elevated tumour necrosis factor-alpha (TNF-alpha) biological activity in psoriatic skin lesions. Clin Exp Immunol. 1994;96:146-151.

5. Leonardi CL, Powers JL, Matheson RT, et al; Etanercept Psoriasis Study Group. Etanercept as monotherapy in patients with psoriasis. N Engl J Med. 2003;349:2014-2022.

6. Lin J, Ziring D, Desai S, et al. TNFalpha blockade in human diseases: an overview of efficacy and safety. Clin Immunol. 2008;126:13-30.

7. Gardam MA, Keystone EC, Menzies R, et al. Anti-tumour necrosis factor agents and tuberculosis risk: mechanisms of action and clinical management. Lancet Infect Dis. 2003;3:148-155.

8. Smith MY, Attig B, McNamee L, Eagle T. Tuberculosis screening in prescribers of anti-tumor necrosis factor therapy in the European Union. Int J Tuberc Lung Dis. 2012;16:1168-1173.

9. Mack U, Migliori GB, Sester M, et al; TBNET. LTBI: latent tuberculosis infection or lasting immune responses to M. tuberculosis? ATBNET consensus statement. Eur Respir J. 2009;33:956-973.

10. Guidelines on the Management of Latent Tuberculosis Infection. Geneva: World Health Organization; 2015. Available from: http://www. ncbi.nlm.nih.gov/pubmed/25973515. Accessed on April 01, 2015.

11. National Technic Steering Group Of The Epidemiological Sampling Survey For Tuberculosis, Duanmu H. [Report on fourth national epidemiological sampling survey of tuberculosis]. Zhonghua Jie He He Hu Xi Za Zhi. 2002;25:3-7. Chinese.

12. Diel R, Wrighton-Smith P, Zellweger JP. Cost-effectiveness of interferon-gamma release assay testing for the treatment of latent tuberculosis. Eur Respir J. 2007;30:321-332.

13. Simsek H, Alpar S, Ucar N, et al. Comparison of tuberculin skin testing and T-SPOT.TB for diagnosis of latent and active tuberculosis. Jpn J Infect Dis. 2010;63:99-102.

14. Tuberculosis. Factsheet $\mathrm{N}^{\circ} 104$ [webpage on the Internet]. Geneva: World Health Organization; 2015. Available from: http://www.who. int/mediacentre/factsheets/fs104/en/. Accessed April 01, 2015.
15. HuiZhang, Lixia Wang. Progress and prospects of tuberculosis control in China. Journal of Tuberculosis and Lung Health. 2013;2:141-142. Chinese. Available from: http://wanfang.jsinfo.gov.cn/D/Periodical_ jhyfbjbzz201303001.aspx. Accessed April 01, 2015.

16. Dixon WG, Hyrich KL, Watson KD, Lunt M, Galloway J, Ustianowski A; B S R B R Control Centre Consortium, Symmons DP; BSR Biologics Register. Drug-specific risk of tuberculosis in patients with rheumatoid arthritis treated with anti-TNF therapy: results from the British Society for Rheumatology Biologics Register (BSRBR). Ann Rheum Dis. 2010;69:522-528.

17. Tubach F, Salmon D, Ravaud P, et al; Research Axed on Tolerance of Biotherapies Group. Risk of tuberculosis is higher with anti-tumor necrosis factor monoclonal antibody therapy than with soluble tumor necrosis factor receptor therapy: the three-year prospective French Research Axed on Tolerance of Biotherapies registry. Arthritis Rheum. 2009;60:1884-1894.

18. Hsia EC, Cush JJ, Matteson EL, et al. Comprehensive tuberculosis screening program in patients with inflammatory arthritides treated with golimumab, a human anti-tumor necrosis factor antibody, in Phase III clinical trials. Arthritis Care Res (Hoboken). 2013;65:309-313.

19. Gómez-Reino JJ, Carmona L, Angel Descalzo M; Biobadaser Group. Risk of tuberculosis in patients treated with tumor necrosis factor antagonists due to incomplete prevention of reactivation of latent infection. Arthritis Rheum. 2007;57:756-761.

20. [No authors listed]. Targeted tuberculin testing and treatment of latent tuberculosis infection. American Thoracic Society. MMWR Recomm Rep. 2000;49:1-51.

21. Perlmutter A, Mittal A, Menter A. Tuberculosis and tumour necrosis factor-alpha inhibitor therapy: a report of three cases in patients with psoriasis. Comprehensive screening and therapeutic guidelines for clinicians. Br J Dermatol. 2009;160:8-15.

22. Carmona L, Gómez-Reino JJ, Rodríguez-Valverde V, et al; BIOBADASER Group. Effectiveness of recommendations to prevent reactivation of latent tuberculosis infection in patients treated with tumor necrosis factor antagonists. Arthritis Rheum. 2005;52:1766-1772.

23. Aziz MA, Wright A, Laszlo A, et al; WHO/International Union Against Tuberculosis And Lung Disease Global Project on Anti-tuberculosis Drug Resistance Surveillance. Epidemiology of antituberculosis drug resistance (the Global Project on Anti-tuberculosis Drug Resistance Surveillance): an updated analysis. Lancet. 2006;368:2142-2154.

24. Expert group of TB prevention and management. [Expert advice on TB prevention and management before initiating treatment with infliximab]. Chinese Internal Medicine. 2009;48:980-982. Chinese. Available from: http://wanfang.jsinfo.gov.cn/D/Periodical_zhnk200911031.aspx. Accessed April 01, 2015.
Drug Design, Development and Therapy

\section{Publish your work in this journal}

Drug Design, Development and Therapy is an international, peerreviewed open-access journal that spans the spectrum of drug design and development through to clinical applications. Clinical outcomes, patient safety, and programs for the development and effective, safe, and sustained use of medicines are a feature of the journal, which

\section{Dovepress}

has also been accepted for indexing on PubMed Central. The manuscript management system is completely online and includes a very quick and fair peer-review system, which is all easy to use. Visit http://www.dovepress.com/testimonials.php to read real quotes from published authors. 